\title{
Does estimator choice influence our ability to detect changes in home-range size?
}

\author{
Johannes Signer ${ }^{1 *}$, Niko Balkenhol ${ }^{1}$, Mark Ditmer $^{2}$ and John Fieberg ${ }^{2}$
}

\begin{abstract}
Background: Estimates of home-range size are frequently used to compare areal requirements of animals over time or space. Comparative studies of home-range estimators have highlighted extreme differences among general classes of methods (e.g., polygon-based and kernel density-based estimators) and sensitivity to the choice of various tuning parameters (e.g., amount of smoothing). These studies, however, have largely failed to consider how estimates of home-range size are typically used in applied research. We illustrate simulation-based methods for comparing estimators, which focus on relative differences in home-range size (over time or space), rather than their absolute magnitude. We also consider Global Positioning Technology (GPS) location data from a black bear (Ursus americanus) from northwestern Minnesota, USA, to illustrate the relevance to real-world data applications.
\end{abstract}

Results: In our examples, estimates of home-range size often differed considerably in absolute magnitude. Yet, for relative differences, the choice of home-range estimator was often negligible. Furthermore, choosing the right estimator was less important than other aspects of study design (e.g., number of animals followed).

Conclusion: Many questions in ecology focus on changes in space-use patterns (over space or time). For these types of questions, home-range estimators should be evaluated in terms of their ability to detect these spatial and temporal patterns. More importantly, home-range estimation should be seen as a means to an end-i.e., estimators provide indices useful for addressing interesting biological questions or hypotheses-rather than as an end to itself.

Keywords: Movement cost, Changes in home-range size, Estimator choice

\section{Background}

Animals interact with conspecifics and their environment, leading to non-random patterns of space-use [1]. Several different analytical methods have been proposed for quantifying these patterns, including home-range estimation (e.g., [2, 3]), habitat and step selection models (e.g., $[4,5])$, and Bayesian state-space models that fit a mixture of random walks to movement data (e.g., [6, 7]). Whereas the latter two approaches often require custom written code and fine tuning to fit a specific data set, a variety of off-the-shelf home-range estimators can be easily implemented in multiple software platforms ( $\mathrm{R}$, ArcGIS, etc.).

Because of their accessibility, home-range estimators are frequently used to compare space-use patterns for animals

\footnotetext{
*Correspondence: jsigner@gwdg.de

${ }^{1}$ Department of Wild life Science, University of Göttingen, Büsgenweg 3, 37077 Göttingen, Germany

Full list of author information is available at the end of the article
}

living in different landscapes (e.g., $[8,9]$ ) or along spatial gradients (e.g., [10]). With the increase of fine-scale spatio-temporal data afforded by Global Positioning Technology (GPS), short-term (weekly, monthly) estimates of home-range size are now also commonly used to explore changes in space-use patters over time (e.g., $[3,11,12])$.

When using home-range estimators to summarize space-use patterns, it is important not to conflate these statistics with the biological concept of an animal's home range [13-15], defined by Burt [16], as "That area traversed by an individual in its normal activities of food gathering, mating, and caring for young. Occasional sallies outside the area, perhaps exploratory in nature, should not be considered part of the home range". An animal's home range is arguably best thought of in terms of cognitive maps, a complex summary of various spatio-temporal utility surfaces representing different resources (e.g., food, shelter, escape routes, or mating and breeding areas), which the animal actively updates as it interacts with its 
environment $[15,17]$. Home-range estimators, by contrast, provide simple, often static summaries of space-use in terms of a boundary on a map, an area, or a probability distribution of the relative time spent in different areas. An estimate of home-range size is best viewed as an index of space-use or movement cost to meet an individual's needs, a response measure that can be related to other measured covariates in order to gain insights into how animals interact with their environment or other organisms (e.g., habitat types and configurations, waterways, urban areas, or other GPS-tagged individuals).

\section{Methods}

\section{Simulation model}

We use a spatially explicit, individual-based model to simulate animal movement. The simulated animal moves in discrete time steps, $t_{1}, \ldots, t_{n}$, in homogeneous (scenario 1 ) or heterogeneous (scenario 2) space. We denote the position of the animal at time step $t$ as vector $\mathbf{x}_{t}=\left(x_{t}, y_{t}\right)$ containing the $x$ and $y$ coordinates at time $t$. At each time step, the animal selects 20 candidate locations indexed with the superscript $j\left(\mathbf{x}_{t+1}^{1}, \ldots, \mathbf{x}_{t+1}^{20}\right)$ for the position at the next time step $(t+1)$. The coordinates for each candidate location $\left(\mathbf{x}_{t+1}^{j}\right)$ are determined by a turning angle $\phi_{j}$ and step length $s_{j}$ from the current position $\left(\mathbf{x}_{t}\right)$. The turning angle is drawn from a wrapped Cauchy distribution with concentration parameter $\rho_{t}$. The step length is drawn from an exponential distribution with rate $\lambda_{t}$. Next the animal assesses the quality of each candidate location, $\mathbf{x}_{t+1}^{j}$ in terms of habitat quality at $\mathbf{x}_{t+1}^{j}$ and the euclidean distance between $\mathbf{x}_{t+1}^{j}$ and $\mathbf{x}_{0}$-the home-range center-resulting in a vector of weights for each candidate location. Weights for habitat quality and distance to home-range center are then summed, resulting in a single weight, $\omega_{t+1}^{j}$, for each candidate location for the position of the animal at the time $t+1$. The animal then chooses one of the candidate locations randomly, with probability proportional to the weights $\omega_{1}, \ldots, \omega_{20}$.

Model parametrization for the different simulation scenarios is shown in Table 1 . We used time steps of $5 \mathrm{~min}$ and modeled the individual for 1 year, so that 104,832 movement steps were available for each simulated individual.

\section{Scenarios}

We investigate two different scenarios that reflect two different biological questions. In the first scenario, an animal is tracked for 1 year, and the researcher uses estimates of short-term "home-range" size as an index to quantify how movement costs change over time. In the second scenario, multiple animals in different landscapes are followed, and the researcher uses estimates of home-range size to detect differences in movement costs between landscapes that vary with respect to the proportions of favorable habitat.

In scenario $1 \mathrm{a}$, we assume that the tendency of the animal to move away from its home-range center changes over the course of a year. To model the underlying pattern, we use a rescaled Gaussian density function centered on the middle of the year (panel A in Additional file 1: Figure S1). For scenario $1 \mathrm{~b}$, we add additional sinusoidal noise to the underlying Gaussian density function, to represent changes in movement that occur on smaller time scales (panel B in Additional file 1: Figure S1).

For the second scenario, movement parameters do not vary temporally, but the landscape is heterogeneous. We simulated landscapes with different amounts of favorable habitat, using a modified version of the random

Table 1 Parameter values at time step, $t$, for finding one of 20 candidate locations (upper half) and for weighting the candidate locations based on habitat and distance to the home range center (lower half). Step lengths were drawn from an exponential distribution with rate parameter $\lambda_{t}$, and turning angles from a wrapped Cauchy distribution with concentration parameter $\rho_{t}$. Candidate locations received weights that depended on habitat type, $\omega_{\text {hab }}$ (scenario 2 only). In addition, an exponential distribution with rate parameter, $\theta_{t}$, was used to weight locations based on their distance to the home-range center. For both rates $\theta_{t}$ and $\lambda_{t}, \mu$ and $\sigma$ were set to constant values of 182 and 36.4 , respectively

\begin{tabular}{|c|c|c|c|}
\hline Parameter & Scenario 1a & Scenario $1 \mathrm{~b}$ & Scenario 2 \\
\hline \multicolumn{4}{|c|}{ Determing candidate locations } \\
\hline$\rho_{t}$ & 0.5 & 0.5 & $\begin{array}{l}0.01 \mathbf{x}_{t} \text { in patch } \\
0.8 \mathbf{x}_{t} \text { in matrix }\end{array}$ \\
\hline$\lambda_{t}^{j}$ & $-e^{\frac{(t-\mu)^{2}}{2 \sigma^{2}}}+2$ & $-e^{\frac{(t-\mu)^{2}}{2 \sigma^{2}}}+2-0.04 \sin \left(\frac{0.2 t}{2 \pi}\right)$ & 0.01 \\
\hline \multicolumn{4}{|c|}{ Weighting candidate locations } \\
\hline$\omega_{\text {hab }}$ & 1 & 1 & $\begin{cases}1 & \mathbf{x}_{t} \text { in patch } \\
0.1 & \mathbf{x}_{t} \text { in matrix }\end{cases}$ \\
\hline$\theta_{t}$ & $-0.4 e^{\frac{(t-\mu)^{2}}{2 \sigma^{2}}}+0.5$ & $-0.4 e^{\frac{(t-\mu)^{2}}{2 \sigma^{2}}}+0.5-0.1 \sin \left(\frac{0.2 t}{2 \pi}\right)$ & 0.01 \\
\hline
\end{tabular}

$\mu$ and $\sigma$ are constant with values of 182 and 36.4 , respectively. Step length, s, habitat weight, $\omega_{\text {hab }}$, and concentration parameter, $\rho$, are constant for scenario 1 and vary for scenario 2 . In contrast, $\lambda_{t}$ varies over time following a rescaled Gaussian density function for scenario 1 and is constant for scenario 2 
cluster algorithm [18] as implemented in [19]. The random cluster algorithm generates landscapes based on spatial autocorrelation of habitat patches controlled through a fragmentation parameter $p$ and the expected amount of habitat $A$. We simulated landscapes with different amounts of favorable habitat, $A$, by drawing this value from a uniform distribution between 0.1 and 0.9 (i.e., 10 to $90 \%$ of the landscape consists of favorable habitat), while holding the degree of fragmentation constant $(p=0.3)$. We allowed turning angles to depend on the habitat type (favorable or unfavorable), and we weighted proposed locations according to their habitat type and the distance of each candidate location to the home-range center (Table 1). In favorable habitat, chosen turning angles were less concentrated (this resembles within-patch foraging) than in unfavorable habitat (resembling inter-patch movement).

For both scenarios, we assumed the home-range center to be at $x=50$ and $y=50$, the center of the landscape. If this placed the home-range center in unfavorable habitat, we assigned the home-range center to the closest pixel with favorable habitat. All simulations were programmed and run using the program $R$ [20].

\section{Analysis}

We estimated home ranges for different sampling rates and analysis intervals (only scenario 1). We sampled each realization of the movement path at three different sampling rates (2, 4, and 24 observations per day). We chose these sampling rates to resemble commonly used sampling schedules of real GPS collars. We then split the sampled data into either weekly or monthly analysis intervals and calculated home ranges for each combination of sampling rate and analysis interval. We set a threshold of 20 for the minimum number of relocations required for any combination of sampling rate and analysis interval. Hence, we did not consider weekly home ranges for a sampling rate of 2 observations per day.

We estimated home ranges with the minimum convex polygon (MCP), kernel density estimator (KDE), and local convex hull $(\mathrm{LoCoH})$. Although a variety of more home sophisticated home-range estimators have been developed in recent years, MCPs and KDEs are the simplest and most widely applied approaches $[14,21]$. We also included LoCoH because previous studies (e.g., $[9,22])$ found that home-range estimates from $\mathrm{LoCoH}$ may be more useful for wildlife managers, because of their ability to better detect sharp boundaries. For KDE, we used bivariate normal kernels on a 100 by 100 grid with a resolution of 1 unit. We did not rescale the data before calculating bandwidth, and we used the same bandwidth for both dimensions. We used four different methods for choosing the bandwidth: 1) the reference (href) method [23], 2) least-squares cross validation (hlscv; [24]), 3) plug-inthe-equation (hpi; [25]), and 4) an ad hoc scaling of the reference bandwidth (hscaled; [26]). This last approach attempts to find the smallest bandwidth that results in a contiguous home-range area. For least-squares cross validation and ad hoc scaling, we performed a grid search with values ranging from $0.1 \times$ reference bandwidth to $2 \times$ reference bandwidth. For least-squares cross validation, we searched for a global minimum. If the algorithm did not converge (this only happened once for scenario $1 b)$, we excluded the simulation run from the analysis. With the LoCoH approach, we used the type $k$ method, with $k$ determined by the square root of the number of fixes. In all cases, we estimated the area associated with the $95 \%$ isopleth. All home-range analyses were implemented using program R (Version 3.1.1) [20] and the $r h r$ (1.0.010) package [27].

For scenario 1, we explored, graphically, temporal patterns in home-range estimates and their variability across multiple simulated data sets for each unique combination of sampling rate, home-range estimator, and analysis interval. Since we were particularly interested how home ranges can be used as a proxy to quantify movement costs, we also looked at differences in home-range size, i.e., the numerical first derivative: $\frac{\text { home-range size }_{t}-\text { home-range size }_{t-1}}{\Delta t}$ versus $t$. We calculated $\frac{\Delta \text { home-range size }}{\Delta t}$ for each realization and then averaged these values at each time point. In order to investigate the variance of different home-range estimators, we calculated the difference between the 97.5 percentile and 2.5 percentile of the home-range size and $\frac{\Delta \text { home-range size }}{\Delta t}$ sampling distribution, and used the width of this interval as a measure of estimator precision at each time point.

For scenario 2 , we sampled $n=(5,10, \ldots, 100)$ individuals from the population and fit linear models relating $\log$ (home-range size) to the amount of favorable habitat, A, $\log$ (home-range size $)=\beta_{0}+\beta_{1} A$. Then, we repeated this process for $R=100$ times for each home-range estimator, sampling rate, and sampling size $n$. We then calculated the interquartile range and the difference of the 97.5 percentile and 2.5 percentile of the sampling distribution for each combination of sample size, sampling rate, and estimator, and again used the widths of these intervals as a measure of estimator precision. For $\beta_{1}$, the slope, we calculated for each estimate a $95 \%$ confidence interval and looked at the number of times we obtained significant results (i.e., the confidence interval did not overlap 0 ).

\section{Bear data}

During May-July, 2007-2011, we captured American black bears living in northwestern Minnesota, USA, using baited barrel traps as part of a larger study investigating 
how bears utilize an agriculturally dominated and fragmented landscape [28]. We immobilized bears using a combination of ketamine hydrochloride and xylazine, or premixed tiletamine hydrochloride and zolazepam (Telazol ${ }^{\circ}$, Elkins-Sinn, Cherry Hill, NJ, USA). Bears were fit with either collars with store-on-board GPS devices or GPS collars using the Iridium satellite system as a data link. During annual winter den visits, we adjusted collar fit and downloaded data from collars. Bear handling was approved by the University of Minnesota's Institutional Animal Care and Use Committees (permit no. 1002A77516). For the analyses presented, we chose to utilize data from a representative 8-year-old female bear without cubs of the year. This individual was outfitted with a GPS collar from Vectronic Aerospace (Berlin, Germany) throughout 2012. We chose this bear because of the complete year of data she provided (den exit to den entrance), frequent fix schedule (fixes attempted every $2 \mathrm{~h}$ ), and high level of home-range fidelity (based on 5 years of GPS-collar data). We used 1711 successful GPS-collar locations (mean time step $=2.54 \mathrm{~h}, \mathrm{SD}$ $=1.17 \mathrm{~h}$ ) from 30 April 2012 to 28 October 2012 . We used 1-, 2-, 4-, and 8-week analysis intervals and estimated the home-range size using the same homerange estimators as were used in the scenario analyses (see above).

\section{Results}

Scenario $1 \mathrm{a} / \mathrm{b}$ : modeling temporally changing movement patterns

For scenario 1a, absolute magnitude of home-range size differed (Fig. 1), with KDE methods producing larger home-range size than MCP and LoCoH. Yet, all estimators captured the underlying temporal pattern. Further, estimates of changes in home-range size, $\left(\frac{\Delta \text { home-range size }}{\Delta t}\right)$, were nearly indistinguishable (Fig. 1), although the magnitude of changes depended somewhat on the analysis interval (Fig. 1), with longer analysis intervals resulting in estimated rates of change that were less variable and smaller in absolute magnitude. Results for scenario $1 \mathrm{~b}$ were similar (to scenario 1a), but only short analysis intervals (weekly) were able to recover the signal of the short-term sinusoidal variation of changes of space requirements (Additional file 1: Figure S7).

The variability of home-range size estimates was dependent on the estimator and the time of the year (Fig. 2). For weekly analysis intervals, only two estimators, KDE (hscaled) and $\mathrm{LoCoH}$, had relatively constant variability (i.e., the width of the 95 percentile interval of the sampling distribution did not depend on the time). For all other estimators and analysis intervals, the width of the 95 percentile of the sampling distribution varied with time, with estimates exhibiting more variability in the middle of the

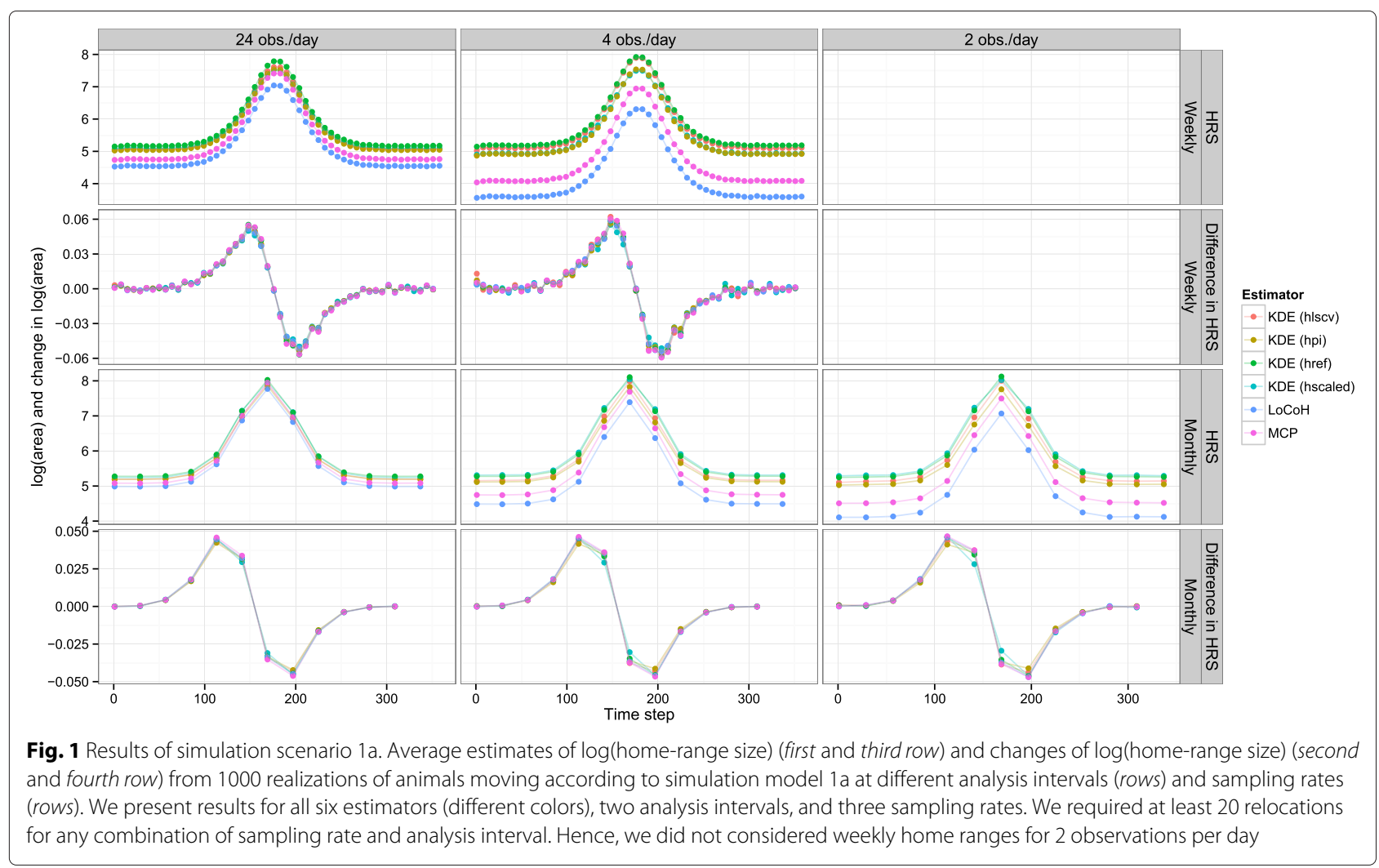




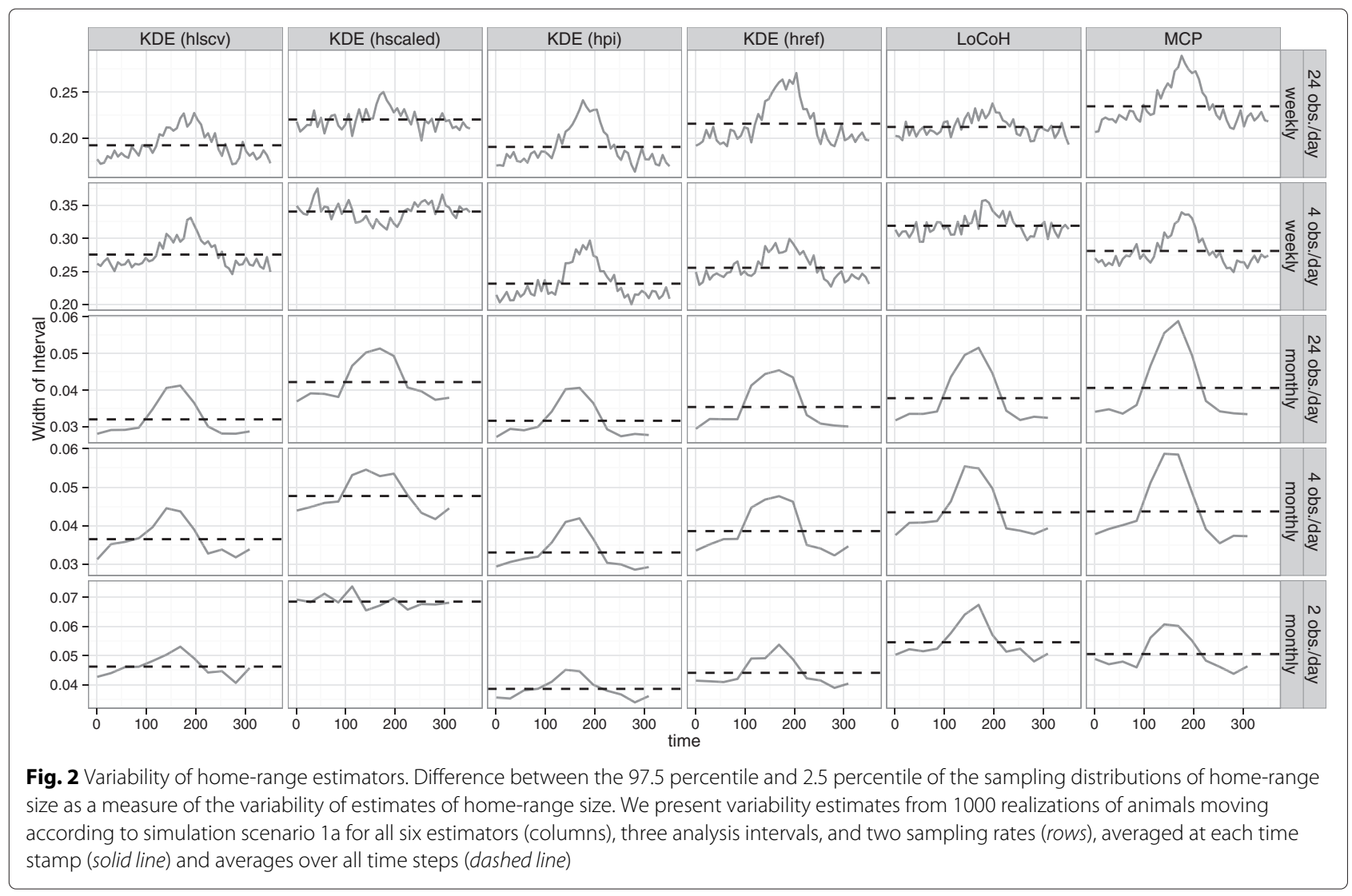

year when animals moved more extensively. Across the range of simulation scenarios (and averaging over time), the variability was smallest for the KDE (hpi) and largest for the KDE (hscaled). Estimates of home-range size (and also rates of change) were less variable when using longer time intervals and when sampling more frequently (Fig. 2 and Additional file 1).

\section{Scenario 2: modeling effects of habitat fragmentation on movement patterns}

Intercepts associated with the linear model relating $\log$ (home-range size) to the amount of favorable habitat differed in magnitude, again suggesting absolute estimates of home-range size varied with estimator choice (Fig. 3). For small sample sizes $(n=5,10)$, the sampling distributions of intercepts $\left(\beta_{0}\right)$ overlapped, but differences among estimators became more pronounced as more individuals were followed. Intercepts associated with KDE (hlscv) and KDE (hpi) were generally smaller than MCP, KDE (href), and KDE (hscaled), with LoCoH falling in between (Fig. 3). Estimates of $\beta_{1}$, which summarizes the influence of habitat on home-range size, were closer to 0 for KDE (lscv) and KDE (hpi); estimates of $\beta_{1}$ were similar in magnitude (and larger in absolute value) for all other estimators (Fig. 3 and Additional file 1).
Precision, and thus the power to detect an influence of the amount of favorable habitat on movement costs, depended mainly on the number of animals followed but was also influenced to a lesser extent by the sampling rate (Figs. 3 and 4).

At the highest sampling rates (24 relocations per day), all estimators had a high power to detect an effect of habitat on home-range size (Fig. 3). With lower sampling rates (e.g., 2 relocations per day), however, many more animals were necessary to detect significant effects, and the choice of estimator becomes more important. In particular, KDE (lscv, hpi) had lower power to detect an effect of habitat than other methods (Fig. 4), largely because the sampling distributions of these two estimators were centered on values closer to 0 (Fig. 3).

\section{Bear data}

We observed a similar, although noisier pattern, with real data originating from a female bear. Absolute home-range size was small in the beginning and the end of the year (this is when bears exit (spring) and prepare to enter (fall) the den). During summer, energy demands increase and bears expand their range to meet their daily needs. All of the estimators captured this trend, but the estimates were of different magnitudes. $\mathrm{LoCoH}$ estimates were the most 


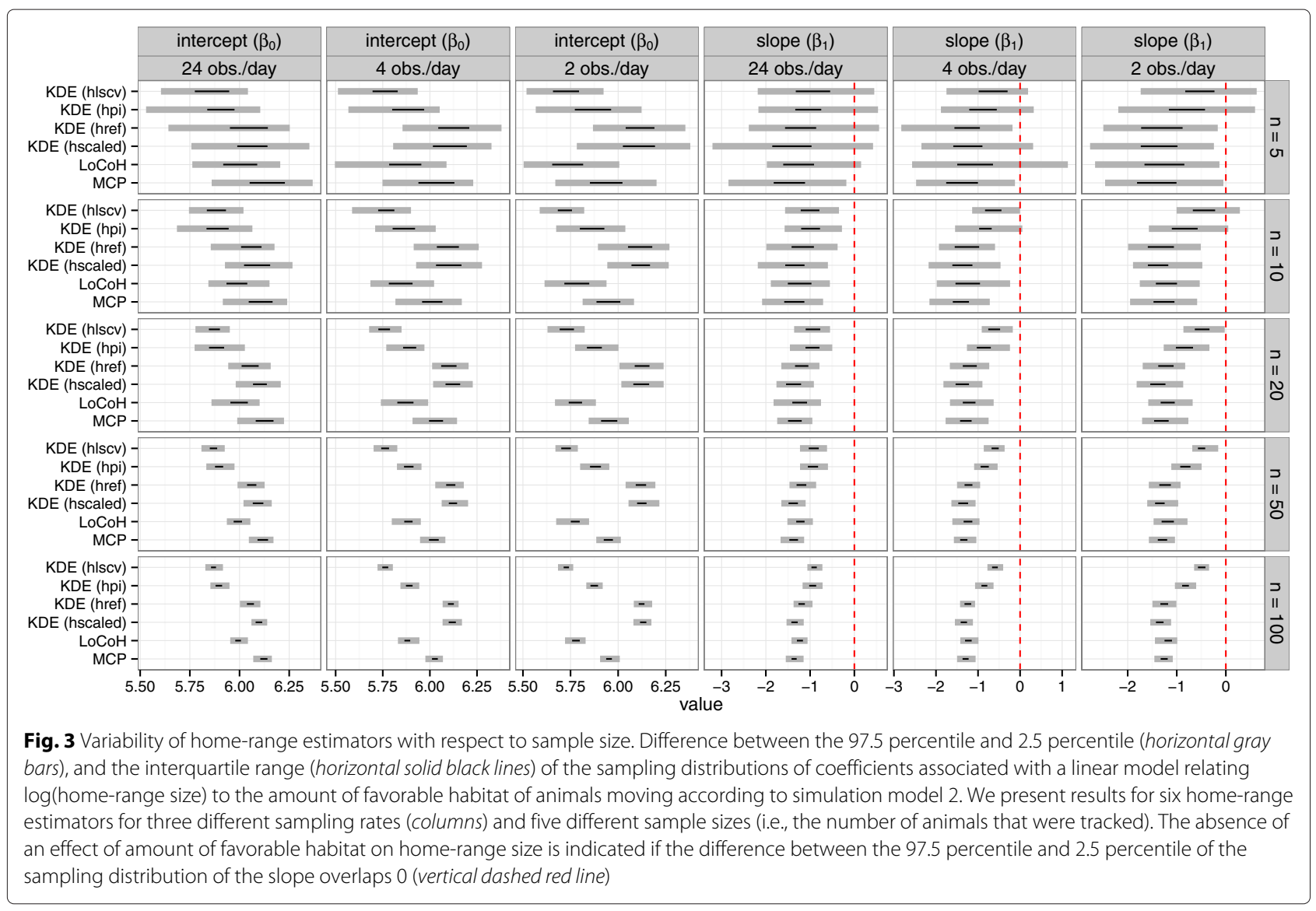

conservative (i.e., smallest), and KDE (href) estimated the largest home ranges (but see also the top panel of Fig. 5). Similar to the simulated data from scenario $1 \mathrm{a}$, differences between estimators became negligible when we evaluated changes in the amount of space used over time (bottom panel of Fig. 5).

\section{Discussion}

In the introduction, we suggested that it could be advantageous, from a statistical and conceptual point of view, to think about home-range estimators as an index of an animal's areal requirement or movement costs. In such cases, the absolute magnitude of home-range size is often less important than how estimates of home-range size change over space and/or time. Importantly, we found that although estimates of home-range size often differed considerably in absolute magnitude, for relative differences, the choice of home-range estimator was often negligible. Furthermore, results of simulation scenario 2 suggest that choosing the right estimator is often less important than

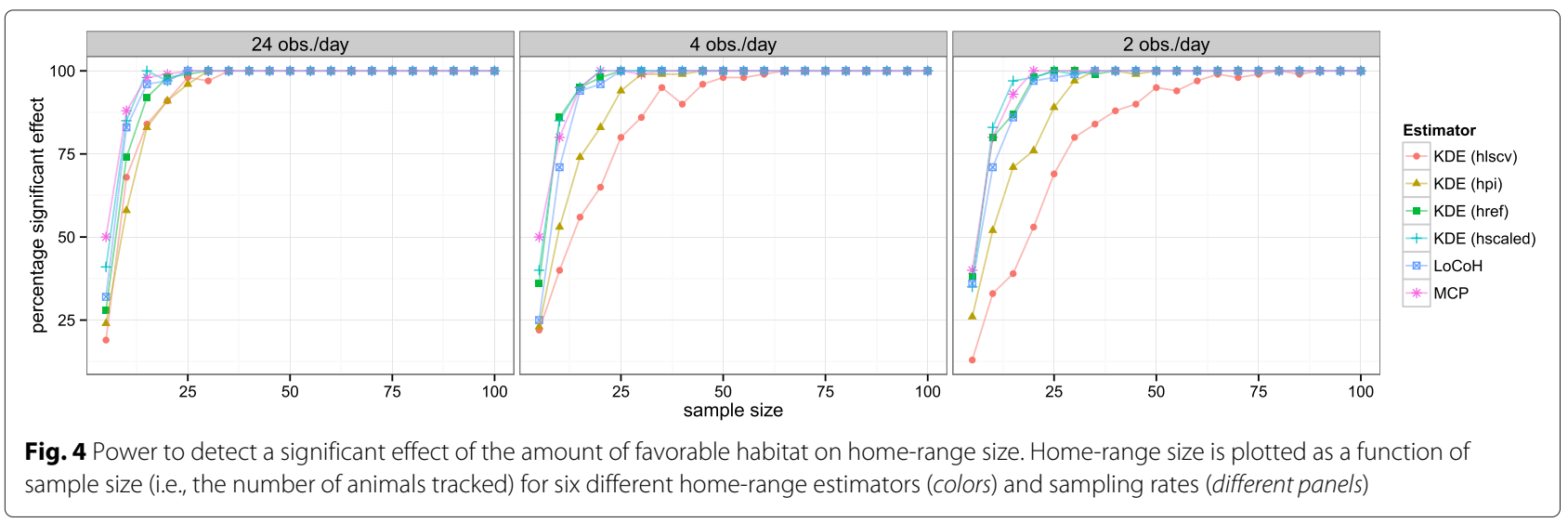



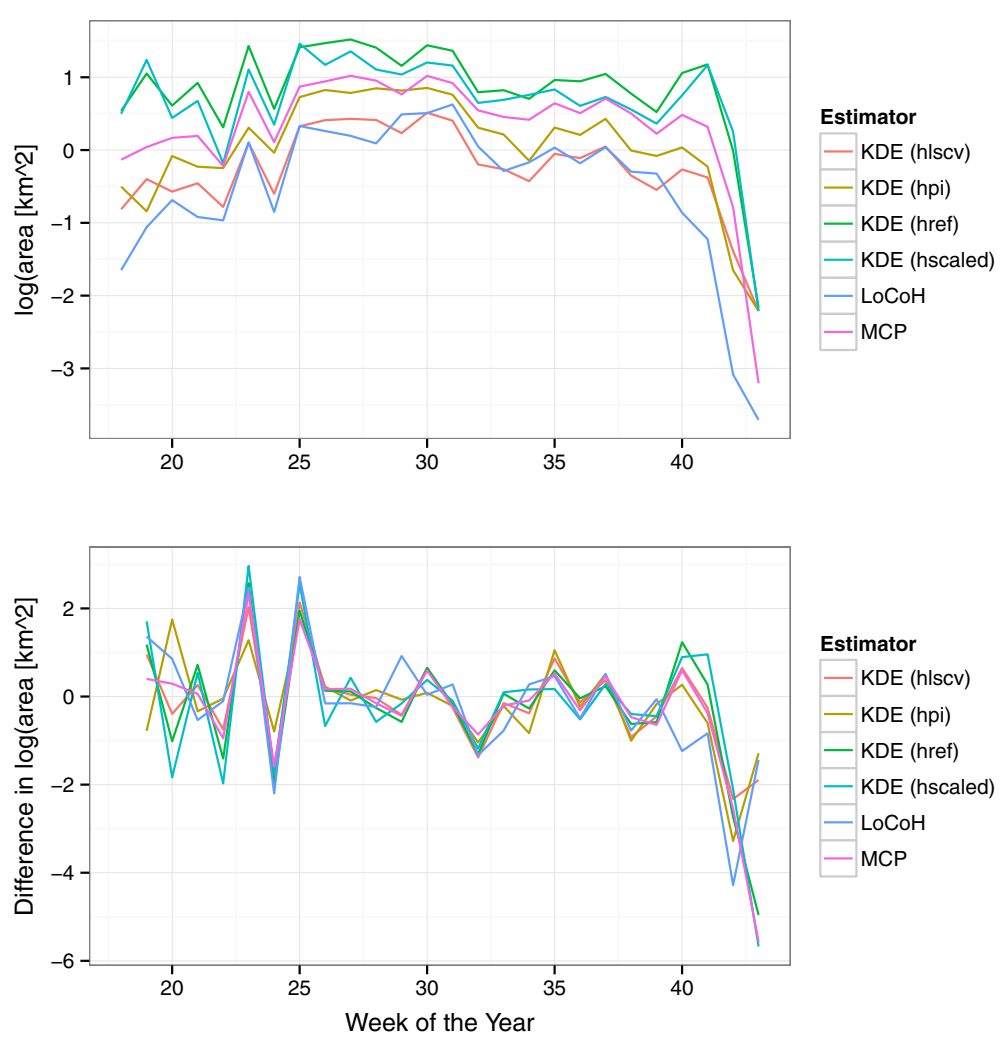

Fig. 5 Results from empirical data. Estimates of home-range size (top panel) and changes of home-range size (bottom panel) with six different home-range estimators over time of a female black bear from northwestern Minnesota

other aspects of study design (e.g., number of animals followed; see also [29]).

Other studies have shown that results may be robust to the choice of home-range estimator (e.g., $[12,30]$ ). Van Beest et al. [12] found that different home-range estimators (KDE and $\mathrm{LoCoH}$ ) provided qualitatively similar results (i.e., the direction of the relationship did not depend on the home-range estimator) regarding the most important factors influencing variation in home-range size across different spatiotemporal scales for moose (Alces alces) in Norway. Also, Nilsen et al. [30] were able to draw the same biological conclusions relating to homerange size from MCP- and KDE-based estimators to body mass for interspecific comparative studies. In congruence with Börger et al. [29], our results suggest that efforts should be directed towards collaring more individuals at the expense of sampling rate; results from scenario 2 highlight this point.

Although comparative studies of home-rage estimators are common (e.g., [24, 31, 32]), most simulation studies tend to focus on bias associated with estimates of home-range size, which requires that one defines a true home range, a concept that can be difficult to quantify using only (real or simulated) animal locations [15, 33].
For many questions, we suggest that a focus on variability of home-range estimates under different circumstances will lead to more informative comparisons. The simulation conducted under scenario 1 would lead us to KDE (hpi) because it was the most precise estimator. By contrast, the power analysis aimed at detecting the influence of habitat on resource needs would lead us to suggest any method except KDE (hpi or hlscv). These conflicting results highlight the need to begin with clearly defined research questions and goals prior to choosing an appropriate home-range estimation method [13].

Our results suggest that for questions like the following: do male individuals require more space than females? does latitude or elevation influence the space-use of a species?, or do individuals of a given species need more space in summer than in winter?, the choice of the estimator will likely be less important than emphasized in the literature while other decisions a researcher might make (e.g., sample size, sample rate, or analysis interval) are at least as important as the estimator choice. Yet, because different estimators give different absolute estimates of home-range size, it is still important, whenever possible, to use the same home-range estimator to analyze all location data. Further, given the differences in implementation 
of home-range estimators (e.g., variability in how $\mathrm{KDE}$ (lscv) is implemented [21]), it is also important to use the same software whenever possible. As a corollary, we strongly encourage researchers to archive raw location data (e.g., in archives like Movebank or dryad) to facilitate meta-analytic hypotheses testing of broad-scale ecological patterns.

Although some research question may require absolute estimates of home-range size, most often, home-range estimates are used to explore changes in space-use patterns over time or space. In these cases, home-range estimators should be evaluated in terms of their ability to detect these patterns. More importantly, home-range estimation should be seen as a means to an end, i.e., estimators provide indices useful for addressing interesting biological questions or hypotheses - rather than as an end to itself [13].

\section{Conclusion}

An advantage of viewing home-range estimates as indices is that it emphasizes the need to start with an interesting and meaningful biological question or hypothesis, rather than viewing home-range estimation as an end in itself [13]. It also suggests that we need to think differently about comparative studies of home-range estimators. We should not only compare the ability of estimators to capture (or exclude) particular areas of space used (or not used) by an animal but also increase emphasis on whether the estimator does a good job of capturing an important biological signal in the data that is relevant to an underlying question or hypothesis of interest. A biased estimator for home-range size may still prove useful if it does a good job of detecting changes in home-range size. For many research questions, this will mean that home-range size is often expected to be proportional to movement cost.

The aim of this manuscript is to illustrate these points by investigating a property of common home-range estimators, the area, through a simulation study. We consider two different simulation scenarios capturing two broadly defined research questions. In the first scenario, we are interested in detecting changes in the amount of space used by an individual over time. In the second scenario, we are interested in comparing the amount of space used by different individuals living in landscapes with different amounts of favorable habitat. In both cases, we simulate animal movement using discrete time steps and calculate home ranges using different estimators, sampling rates, and analysis intervals (scenario 1 only; movement behavior did not change over time for scenario 2). In both cases, it is natural to ask, "What home-range estimator is most appropriate for detecting differences in the amount of space used (changes over time in scenario 1 or differences among landscapes with different amounts of habitat in scenario 2)." In addition, we ask "What analysis interval is most appropriate for exploring temporal space-use patterns?" (scenario 1 only). We explore these questions using simulated data, but we also consider GPS location data from a black bear (Ursus americanus) inhabiting northwestern Minnesota, USA, to illustrate the relevance of our simulation results, and method of estimator comparison to real-world data applications.

\section{Additional file}

Additional file 1: Results of modeling scenarios that were not presented in the main text.

Competing interests

The authors declare that they have no competing interests.

\section{Authors' contributions}

JS perfomed the modeling and performed the statistical analysis together with JF. The bear movement data was provided by MD. All authors contributed to the interpretation of results and the writing. All authors read and approved the final manuscript.

\section{Acknowledgements}

We thank R. Powell and one anonymous reviewer for the helpful comments on the manuscript. We also thank the Wildlife Discussion Group at the Göttingen University for the valuable feedback on the manuscript.

\section{Author details}

${ }^{1}$ Department of Wildlife Science, University of Göttingen, Büsgenweg 3, 37077 Göttingen, Germany. ${ }^{2}$ Department of Fisheries, Wildlife, Conservation Biology University of Minnesota, St. Paul, USA.

Received: 26 February 2015 Accepted: 15 April 2015

Published online: 01 July 2015

\section{References}

1. Börger L, Dalziel BD, Fryxell JM. Are there general mechanisms of animal home range behaviour? A review and prospects for future research. Ecol Lett. 2008;11(6):637-50. doi:10.1111/j.1461-0248.2008.01182.x.

2. Anderson DP, Forester JD, Turner MG, Frair JL, Merrill EH, Fortin D, et al. Factors influencing female home range sizes in elk (Cervus elaphus) in north american landscapes. Landsc Ecol. 2005;20(3):257-71.

3. Börger L, Franconi N, Ferretti F, Meschi F, De Michele G, Gantz A, et al. An integrated approach to identify spatiotemporal and individual-level determinants of animal home range size. Am Nat. 2006;168(4):471-85.

4. Manly BF, McDonald LL, Thomas DL, McDonald TL, Erickson WP. Resource selection by animals: statistical design and analysis for field studies. Dordrecht, The Netherlands: Kluwer Academic Publishers; 2002.

5. Thurfjell H, Ciuti S, Boyce MS. Applications of step-selection functions in ecology and conservation. Mov Ecol. 2014;2(1):4. doi:10.1186/2051-3933-2-4.

6. Patterson T, Thomas L, Wilcox C, Ovaskainen O, Matthiopoulos J. State-space models of individual animal movement. Trends Ecol Evol. 2008;23(2):87-94. doi:10.1016/j.tree.2007.10.009.

7. McClintock BT, King R, Thomas L, Matthiopoulos J, McConnell BJ, Morales JM. A general discrete-time modeling framework for animal movement using multistate random walks. Ecol Monogr. 2012;82(3): 335-49. doi:10.1890/11-0326.1

8. Walter WD, VerCauteren KC, Campa III H, Clark WR, Fischer JW, Hygnstrom SE, et al. Regional assessment on influence of landscape configuration and connectivity on range size of white-tailed deer. Landsc Ecol. 2009;24(10):1405-1420.

9. Reinecke H, Leinen L, Thißen I, Meißner M, Herzog S, Schütz S, et al. Home range size estimates of red deer in Germany: environmental, individual and methodological correlates. Eur J Wildl Res. 2013;60(2): 237-47. doi:10.1007/s10344-013-0772-1. 
10. Morellet N, Bonenfant C, Börger L, Ossi F, Cagnacci F, Heurich M, et al. Seasonality, weather and climate affect home range size in roe deer across a wide latitudinal gradient within Europe. J Anim Ecol. 2013;82(6): 1326-1339. doi:10.1111/1365-2656.12105.

11. Knight CM, Kenward RE, Gozlan RE, Hodder KH, Walls SS, Lucas MC. Home-range estimation within complex restricted environments: importance of method selection in detecting seasonal change. Wild Res. 2009;36(3):213-24.

12. van Beest FM, Rivrud IM, Loe LE, Milner JM, Mysterud A. What determines variation in home range size across spatiotemporal scales in a large browsing herbivore? J Anim Ecol. 2011;80(4):771-85. doi:10.1111/j. 1365-2656.2011.01829.x.

13. Fieberg J, Börger L. Could you please phrase "home range" as a question? J Mammal. 2012;93(4):890-902.

14. Kie JG, Matthiopoulos J, Fieberg J, Powell RA, Cagnacci F, Mitchell MS, et al. The home-range concept: are traditional estimators still relevant with modern telemetry technology? Phil Trans R Soc B: Biol Sci. 2010;365(1550):2221-231. doi:10.1098/rstb.2010.0093.

15. Powell RA, Mitchell MS. What is a home range? J Mammal. 2012;93(4): 948-58.

16. Burt WH. Territoriality and home range concepts as applied to mammals. J Mammal. 1943;24(3):346-52.

17. Nathan R, Getz WM, Revilla E, Holyoak M, Kadmon R, Saltz D, et al. A movement ecology paradigm for unifying organismal movement research. Proc Natl Acad Sci. 2008;105(49):19052-9.

18. Saura S, Martínez-Millán J. Landscape patterns simulation with a modified random clusters method. Landsc Ecol. 2000;15(7):661-78.

19. Efford M. Secr: spatially explicit capture-recapture models. 2013. R package version 2.7.0. http://cran.r-project.org/package=secr.

20. R Core Team. R: a language and environment for statistical computing. Vienna, Austria: R Foundation for Statistical Computing; 2013. http://www.r-project.org.

21. Laver PN, Kelly MJ. A critical review of home range studies. J Wildl Manag. 2008;72(1):290-8. doi:10.2193/2005-589.

22. Scull P, Palmer M, Frey F, Kraly E. A comparison of two home range modeling methods using Ugandan mountain gorilla data. Int J Geogr Inf Sci. 2012;26(11):2111-121. doi:10.1080/13658816.2012.691507.

23. Worton B. Kernel methods for estimating the utilization distribution in home-range studies. Ecology. 1989;70(1):164-8.

24. Seaman DE, Powell RA. An evaluation of the accuracy of kernel density estimators for home range analysis. Ecology. 1996;77(7):2075-085.

25. Sheather SJ, Jones MC. A reliable data-based bandwidth selection method for kernel density estimation. J R Stat Soc Ser B Methodol. 1991;53:683-90.

26. Kie JG. A rule-based ad hoc method for selecting a bandwidth in kernel home-range analyses. Anim Biotelemetry. 2013;1(1):1-12.

27. Signer J, Balkenhol N. Reproducible home ranges ( $r$ hr ): a new, user-friendly R package for analyses of wild life telemetry data. Wildl Soc Bull. 2015. doi:10.1002/wsb.539.

28. Ditmer MA. American black bears: strategies for living in a fragmented, agricultural landscape. PhD thesis, University of Minnesota, Saint Paul, Minnesota 2014.

29. Börger L, Franconi N, De Michele G, Gantz A, Meschi F, Manica A, et al. Effects of sampling regime on the mean and variance of home range size estimates. J Anim Ecol. 2006;75(6):1393-405.

30. Nilsen EB, Pedersen S, Linnell JDC. Can minimum convex polygon home ranges be used to draw biologically meaningful conclusions? Ecol Res. 2007;23(3):635-9. doi:10.1007/s11284-007-0421-9.

31. Lichti NI, Swihart RK. Estimating utilization distributions with kernel versus local convex hull methods. J Wildl Manag. 2011;75(2):413-22. doi:10.1002/jwmg.48.

32. Schuler KL, Schroeder GM, Jenks JA, Kie JG. Ad hoc smoothing parameter performance in kernel estimates of GPS-derived home ranges. Wildl Biol. 2014;20(5):259-66. doi:10.2981/wlb.12117.

33. Fieberg J. Kernel density estimators of home range: smoothing and the autocorrelation red herring. Ecology. 2007;88(4):1059-66.

\section{Submit your next manuscript to BioMed Central and take full advantage of:}

- Convenient online submission

- Thorough peer review

- No space constraints or color figure charges

- Immediate publication on acceptance

- Inclusion in PubMed, CAS, Scopus and Google Scholar

- Research which is freely available for redistribution

Submit your manuscript at www.biomedcentral.com/submit
C) Biomed Central 